\title{
Stazio personaggio, poeta e cristiano
}

Il canto 21 del Purgatorio è il primo dei canti in cui è figura centrale Stazio, il poeta latino che Dante conosce per la Tebaide e per l'Achilleide, non per le Silvae, che crede tolosano invece che napoletano, e che ritiene convertito al Cristianesimo anche se la sua fede restò segreta ("chiuso cristian fu'mi", 22.90). In queste notizie che Dante fornisce del suo nuovo personaggio c'è un misto di verità, di informazione erronea e di leggenda, che ha aguzzato l'ingegnosità degli interpreti e li ha spronati a cercare fonti rare, magari note a Dante e non giunte fino a noi per via diretta. Sono state e continuano ad essere ricerche molto interessanti e molto erudite, che però non è sempre facile e utile misurare con la circostanziata realtà del testo dantesco. Il quale fa di Stazio non certamente una guida aggiuntiva a Virgilio, a Beatrice e a San Bernardo, anche se con le tre guide non mancano suoi punti di contatto di varia natura e di vario spessore, come sono l'illustrazione di strutture dell'oltretomba o la spiegazione di problemi filosofici.

Ovviamente, se un confronto con la funzione di Beatrice è più difficilmente proponibile, ben agevole è la somiglianza che si può sottolineare con Virgilio, del quale Stazio si fa compagno insieme a Dante per l'ultimo tratto del cammino verso il Paradiso terrestre: li avvicinano la poesia, la latinità, la conoscenza approfondita a livello del linguaggio e dello stile che Dante ne ha; ciò, poi, che manca a Virgilio e Stazio ha, cioè la fede, e che ha fatto pensare qualche critico ad una sorta di gradazione rappresentata dal secondo nell'avvicinamento a Beatrice, è cosa che mette Stazio piuttosto nella condizione di Dante che non in quella di Virgilio-guida, accomunati dalla possibilità di ottenere un obiettivo unico e anche dalla realizzazione di un'esperienza che vale per ambedue (la liberazione in generale e in particolare l'immersione finale nell'Eunoè).

A San Bernardo invece Stazio pare assomigliare di più nel confronto dei processi strutturali delle due cantiche cui rispettivamente appartengono: come Bernardo fa nel Paradiso dopo che Beatrice ha lasciato Dante per andare a collocarsi nel suo posto all'interno della rosa mistica, che è il luogo dove Dante potrà poi rivederla per l'ultima volta, così Stazio nel Purgatorio accompagna Dante alla presenza di Beatrice. Ma mentre Bernardo resta solo con Dante e assolve cos̀̀ il suo ruolo di guida-la figura, insomma, cui Dante resta affidato come lo scolaro al maestro più diretto-conducendo Dante, appunto, dinanzi allo spettacolo conclusivo dell'Empireo, ed in esso della stessa Beatrice, suggerendogli poi la preghiera alla Vergine e apprestandolo, infine, all'ineffabile contemplazione di Dio, Stazio rimane per il primo, più lungo tratto della sua presenza accanto a 
Dante (canti 21-30) in compagnia di Virgilio e per il secondo (canti 30-33) sotto l'alto patronato di Beatrice, sollecita, sia pure di scorcio, verso di lui nella stessa misura che verso Dante.

Stazio, pertanto, è solo un compagno di viaggio e non una guida, ed è più prossimo a Dante che a Virgilio. Da questi ha ricevuto un sapere mondano, che essendo giusto ed onesto lo ha favorito nella conversione alla vera fede; solo questa però ha consentito ch'egli avesse una condizione diversa, e possa ora arrivare a godere della beatitudine. Per questa ragione potrà dare a Virgilio, ma soprattutto per utilità di Dante, risposte a cose che quest'ultimo non sa per difetto d'esperienza e per intrinseca fallacia del sapere umano. Queste cose si dipanano lungo il 21, il 22 e ancora il canto 25 del Purgatorio, e nell'ordine sono le seguenti.

In 21, su domanda di Virgilio, offre una spiegazione tecnica, ovvero teologica del terremoto che alla fine del canto 20 ha scosso l'intera montagna, dalla quale è salito unanime il coro evangelico $(L c .2 .14)$ delle anime di tutti i penitenti (20.133-38); quindi dichiara per disteso la propria identità, che è quella di poeta debitore in tutto a Virgilio. In 22, ancora a richiesta di Virgilio, spiega che il suo peccato fu di prodigalità e non d'avarizia; di seguito racconta come avvenne la sua conversione al Cristianesimo, sollecitato sì da un testo pagano com'è la quarta ecloga di Virgilio, ma contenente una non oscura profezia d'un novus ordo che sta per discendere sul mondo. Ma sia alla fine del 21 che a quella del 22 è lui, a sua volta, a ricevere illuminazione, insomma risposte a cose che ignora, una volta da Dante sull'identità di Virgilio, una volta da Virgilio stesso sulle presenze del Limbo. Nel che è precisamente individuabile un quasi perfetto parallelismo tra i canti 21 e 22, con due domande ciascuno che passano da Virgilio a Stazio e una che passa da Stazio a Dante e da Stazio a Virgilio. Facile dedurne che una stessa ragione retorico-narrativa governa i due canti e dunque un'espansione ordinata dell'episodio, più ordinata almeno di quanto non avvenga tra $\mathrm{i}$ canti 6 e 7 del Purgatorio stesso, singolarmente prossimi, nella nostra memoria di lettori, come $\mathrm{i}$ canti dell'incontro con Sordello, cioè con un altro poeta, che ha da fare, anche se per motivi tutti diversi, con Virgilio. In 25 infine Virgilio lascia a Stazio il privilegio di spiegare a Dante come possa accadere che delle ombre possano soffrire come se avessero il corpo e ne nasce un'intera trattazione della genesi dell'uomo, dell'anima e delle ombre. Sono, queste, cose che per la verità Virgilio sa, al contrario della causa del terremoto, e che anzi è capace di esemplificare su richiami alla mitologia classica, mostrando per una via speciale le possibilità di collegamento che esistono tra universo precristiano e universo cristiano. In ogni caso si assiste ad una crescita della responsabilità intellettuale e quindi morale di Stazio, che è parallela alla crescita della conoscenza e della perfezione di Dante.

Il quale è più prossimo a Stazio, in primo luogo perché come questi è uno spirito in cerca di liberazione dagli ostacoli e dai vincoli del mondo terreno; e in questo senso Stazio è una sorta di "figura" di Dante, in quanto nel "poema sacro" 
precorre quella che sarà in un futuro non tanto lontano l'esperienza di Dante. In secondo luogo, Dante ha in lui un modello di biografia intellettuale-poetica, in quanto Stazio è stato buon discepolo di Virgilio prima di lui, ha insomma compiuto un'esperienza che Dante ha ripetuto. In terzo luogo compie con Dante delle esperienze da vero compagno di viaggio, rinfrescando a Dante la memoria del suo incontro con Virgilio, ripercorrendo con lui, ovvero col racconto fatto a lui, luoghi che ha appena attraversato e soprattutto tuffandosi insieme a lui nelle acque dell'Eunoè (33.134-35), che è il momento culminante di una rete di allusioni della presenza di Stazio accanto a Dante (basti rinviare a 23.8, a 26.17 e a 28.146, particolarmente, là dove Dante, ricevuta da Matelda spiegazione di un dubbio nato dal contrasto con quello che Stazio gli ha appena spiegato, si rivolge "'n dietro allora tutto / a' miei poeti, e vidi che con riso / udito avean l'ultimo costrutto").

Stazio dunque non è una quarta guida di Dante, ma è piuttosto un compagno e un esempio, e lo è su un terreno spirituale, naturale sbocco d'ogni condizione naturale e sovrannaturale, ma ancor prima lo è sul terreno della poesia, che è un particolare modo di manifestarsi del sapere, che è unico, viene dalla stessa origine e tende allo stesso fine. L'ambito cui rinvia, pertanto, il 21 del Purgatorio, unitamente al 22 e in buona misura anche al 25, è quello dell'incontro di Dante con i poeti. È, questa, un'associazione istantanea, che si collega ad un filo rosso che percorre per lunghi tratti e sia pure con sensi e segni diversi la Divina Commedia. Esso si snoda dal primo canto dell'Inferno all'improvvisa apparizione di Virgilio che "per lungo silenzio parea fioco", tanto da consentire un'ipotesi alternativa tra un suo esser "ombra o omo certo". Quando poi si dichiara e si dice ombra, mantovano, vissuto al tempo di Augusto, completa e corona la dichiarazione con la sua condizione di poeta:

Poeta fui, e cantai di quel giusto figliuol d'Anchise che venne di Troia, poi che 'l superbo Ilión fu combusto.

Si badi: è un'identità che si acquisisce solo ed insieme a quella del personaggio che agisce il suo poema. Il quale, a sua volta, come è subito richiamato, è la storia di un viaggio dalla distruzione e dalla morte alla vita e alla fondazione di un nuovo regno in un altro spazio, grande, Latium. Poco più avanti Dante incontra questo stesso personaggio, con altri di opere di letteratura e di quello stesso poema. Il personaggio e il suo autore sono dunque accomunati per sempre nello stesso luogo, ambedue reali per sempre perché sono stati nella storia, sia pure quella dei poemi. La storia infatti è solo il luogo di verifica di verità che la travalicano, è tutta simbolo di altro.

Nel suo spessore e nel suo intrecciarsi, il filo rosso dei poeti e della poesia si propone dunque fin dall'introduzione dell'opera, prima ancora che il viaggio nell'oltretomba si avvii. Un marcatissimo ispessimento lo riceve in Inferno 4 
nell'incontro con gli altri grandi poeti onorati da Dante, Omero, Orazio, Ovidio, Lucano, e nell'aerea, gotica grazia del "nobile castello", che nasconde nel cuore più segreto il "verde smalto" affollato di dotti, di poeti e delle loro creature. Lungo l'Infemo, invece, il filo sembra scolorirsi, anche se non si smarrisce per via d'una serie di segnalazioni o indirette, come nel caso di Guido Cavalcanti attraverso il proprio padre, o dirette, come per Pier della Vigna, Brunetto Latini, Bertran dal Bornio, ma di significazione diversa e non unificabile.

Nel Purgatorio esso raggiunge il massimo di spessore e la coloritura più intensa-nonché una comunicabilità, se non un'unità, di significazione--attraverso la successione d'incontri e di colloqui, disseminati lungo tutta la montagna, con Sordello, Bonagiunta, Forese, Guinizzelli, Daniel, nonché con Stazio, e anche attraverso la salda presenza nella memoria di altri, come ancora i due Guidi (11.97-99) e anche se stesso in una scala che va dall'autocitazione (2.112-14, dove Casella comincia a cantare la canzone del terzo trattato del Convivio, "Amor che ne la mente mi ragiona", e canto 24.51 , dove Bonagiunta richiama la prima canzone della Vita nuova, "Donne ch'avete intelletto d'amore") alla riflessione autobiografica (canto 30 soprattutto, ma un poco anche canto 11, come s'è detto). Si dovrà riflettere da parte nostra, in particolare, su questa maggior presenza di poeti, e quindi di poesia e d'arte, nel Purgatorio rispetto alle aitre cantiche. Specialmente rispetto al Paradiso, dove quel filo veramente si smarrisce, tanto che, anche quando uno che fu poeta viene esaltato nella sua beatitudine, la sua qualità specifica non ha più rilevanza, come accade per Folchetto ( $P a r$. 9). È ovvio che una branca settoriale del sapere si annulli, come ogni altra, nella partecipazione alla conoscenza più alta, quella di Dio. Nel Purgatorio, invece, i poeti contano proprio come poeti oltre che come spiriti in attesa di conseguire una beatitudine già loro assicurata e volentieri parlano della loro esperienza di poeti, espongono graduatorie tra i poeti, discorrono di meriti acquistati, di demeriti e di insufficienze. Nello svolgimento di quel filo rosso poi, proprio i canti di Stazio, il quale prende tanto spazio da ritenersi giustificato talvolta il cercarne una particolare funzione, sono momenti di grande spicco.

Una segnalazione inequivocabile in tale senso dall'interno di questi canti si riceve dalla frequenza dei richiami verbali e concettuali alla poesia, al fare poetico e ai poeti, come ovviamente non può che accadere in canti dove il deuteragonista di un poeta è stato e resta egli stesso poeta. Così di seguito alla perifrasi che costituisce la più alta esaltazione della poesia che da lungo tempo si sia udita nelle letterature occidentali, quella con la quale Stazio stesso si definisce,

col nome che più dura e più onora,

confermandola di seguito con l'allusione-di sapore tutto preumanistico, mussatiano e petrarchesco, come è stato sottolineato e si può agevolmente sottoscrivere-all'incoronazione poetica ottenuta, lui tolosano, a Roma, 
dove mertai le tempie ornar di mirto,

si ha "vocale spirto" (21.88) come metafora di canto, eloquio (tra i commentatori si veda per tutti Sapegno, commento al luogo), cui è allegato un "dolce" che anticipa (e resta poi fermo nella memoria) il "dolce stil novo" della definizione di Bonagiunta in 24.57, anche se nella storia di Dante segue alla definizione dello stesso Stazio quale "poeta dolce" di Conv. 4.25.1, "cantai" (21.92) nel preciso senso di poetai, "poetando" (21.98), ancora "cantar" per poetare in 21.126; e in 22.55 , s'incontra nuovamente "cantasti" e in 57 Virgilio è definito quale "cantor de' bucolici carmi", per la ragione subito di seguito evidente dell'interpretazione medievale corrente della quarta ecloga; e andrà sottolineato il prezioso sintagma "m 'inviasti / verso Parnaso" (22.64-65) e quindi "poeta" (73) in dicotomia speculare con "cristiano", "poetando" (22.89), ancora il richiamo all'incoronazione poetica di vari greci ("che già di lauro omar la fronte", 22.108), "ambedue li poeti", Virgilio e Stazio, che insieme scrutano la parete della montagna in cerca del varco per salire alla comice successiva (22.115), infine "i lor sermoni / ch'a poetar mi davano intelletto" (128-29) e che anche si presentano all'orecchio di Dante quali "dolci ragioni" (130).

È ovvia l'insistenza sempre maggiore che, dopo il parallelismo poesia-cristianesimo, viene a prendere quello di poesia-sapere. "Li due poeti" di 22.139, appressantisi all'albero che coi suoi frutti costituisce l'immagine di pena dei golosi siti nella nuova comice e con le voci che ne discendono richiama luoghi storicopolitici classici e biblici mescolati insieme e culminanti non per caso nella rappresentazione del digiuno di San Giovanni Battista nel deserto (151-54), risulta, così, piena proposizione dell'intrinseco sapere di cui i poeti possono essere portatori, consci o inconsci, quale si dilata nel canto 25, dopo l'ispessirsi del linguaggio strumentale della poesia che si ha negli incontri con Forese e con Bonagiunta. E tuttavia, né in questi canti 23-24, né nel guinizzelliano 26 , si ha un consimile affollarsi delle parole della poesia e sue immediate metafore-come "ardor", "faville", "scalda", "fiamma" (21.94-95).

Si ricordi e si aggiunga infine che Dante riprende e rinnova proprio qui l'elencazione dei poeti e dei loro personaggi varata in Inferno 4; sicché, dopo il ricordo di Giovenale di 22.14, indicato come fonte di quanto avvenuto dopo, ecco una nuova sfilza di poeti latini e greci, da Terenzio (22.97) a Agatone (107) e quindi di personaggi della poesia (110-14).

Col tema dell'incontro con i poeti Dante esplicita dall'interno dell'opera l'importanza ch'egli attribuisce alla poesia nel proprio sistema morale e intellettuale; e col ricorso ai poeti ha modo di enunciare le sue scelte, almeno alcune scelte che ha compiuto in passato su questo terreno e che nella Commedia o conferma o precisa. Che proprio nel Purgatorio egli tratti con maggior ampiezza e profondità questo aspetto dell'organizzazione del pensiero e del sapere è, ovvia- 
mente, da mettere in relazione con la dimensione penitenziale degli spiriti che incontra, come se proprio essa offrisse ad ognuno un maggior agio di spazio e di tempo per riflettere su di sé, sui propri errori e sui propri ravvedimenti ed insieme sulla rettitudine e sulle deviazioni altrui: la condizione di penitente, in quanto presa di coscienza dell'interiorità, consente anche maggior libertà.

È tra queste coordinate che i canti 21 e 22 del Purgatorio e per ragioni diverse, come nel seguito cercherò di mostrare, anche il dottrinale 25 vanno iscritti nel tema di Dante e i poeti. Il che vale a ribadire convinzioni e dichiarazioni già enunciate altrimenti e in altri momenti, come quella importantissima e centralissima della devozione di Dante al suo maestro di poesia e ora anche di esperienza Virgilio; ma serve anche ad un nuovo bilancio di cosa significhi la poesia nell'ordine universale e in quanto manifestazione più alta dell'esprimersi con parole. Questa impostazione obbligata sprona, con conseguente immediatezza, a chiarire la posizione dei canti in questione in una rete di rapporti più vasta. Infatti, nella grande struttura unitaria che è la Divina Commedia essi presentano alcune singolarità sia che si guardi al 'poema sacro' come viaggio verso la redenzione, storia del conseguimento della salvezza attraverso l'esperienza diretta di un universo simbolico ma anche veramente alternativo a quello reale, sia che ci si concentri invece sull'edificio letterario sapientemente costruito col pieno dispiegamento di quelle tecniche che un fascio di tradizioni scrittorie risalenti a\}meno alla classicità latina ha costituito. Resta così enunciata una dicotomia fondamentale, che nel corso dei secoli s'è venuta fissando come una sorta di guida binaria per la lettura d'ogni momento dell'opera, in un parallelismo perfetto che non privilegia un aspetto sull'altro e che si può riassumere nella doppia definizione della Commedia come opus symbolicum e come opus retoricum. Una dicotomia, questa, che se ha il suo fondamento nella distinzione tra i primi due dei quattro sensi che Dante attribuisce alle scritture, cioè il senso letterale e quello allegorico, anche la trascende perché, da una parte, sotto l'idea del simbolo si accolgono anche gli altri due sensi delle scritture secondo Dante, il morale e l'anagogico, mentre, d'altra parte, essa propone indirettamente una dicotomia d'altra natura, più elementare e primaria, per così dire, precisamente di natura linguistica, quella insomma tra significante e significato del segno.

Ma mi si consenta qui una piccola parentesi, forse non del tutto inutile alle note che si stanno svolgendo, sia pure con una incidenza un po' laterale. La dicotomia simbolo/retorica attraverso la teoria dei quattro sensi, Dante l'applica a tutte le scritture, le sacre come le profane. Presiede a questa attribuzione una concezione delle scritture come atto arcano, che in sé nasconde qualche altra cosa, fatto piuttosto per celare che per palesare nel momento stesso del consentire la comunicazione. Scrivere insomma è una traduzione, una messa in cifra del messaggio. A costituire questa nozione della scrittura concorrono molti elementi della tradizione da tardo-antica a tardo-medievale, dal neoplatonismo, ovvero platonismo-agostinismo latente di tutta la cultura medievale, alle dottrine eterodosse ed esoteriche, tra le quali si colloca da ultimo, almeno nella trasmissione 
palese della sua sostanza alla cultura occidentale, la Kabbala. È difficile credere che Dante ignorasse totalmente la Kabbala, che certo non era semplice distinguere pienamente e coscientemente dall'ebraismo ortodosso, ma elementi della quale circolavano certamente al di fuori del suo corpo costituito. Ma è una ricerca tutta da fare e della quale qui non si voleva che segnalare la possibile apertura.

Uscendo di parentesi e tornando alla dicotomia opus symbolicum / opus reioricum a proposito delle singolarità del 21 del Purgatorio, svolgerò qualche considerazione sul secondo elemento della dicotomia in quanto consente un discorso fornito di un referente più immediato, che è il testo nella sua materialità.

Al primo livello della retorica, quello dell'inventio ovvero della favola, da intendersi insieme come contenuto e come forma della narrazione, il 21 del Purgatorio resterebbe immotivato e addirittura incomprensibile senza la conoscenza di quanto è raccontato nella conclusione del canto 20 (127-41): il terremoto che scuote la montagna. Difatti già l'improvvisa apparizione di un'ombra che si accoda ai due pellegrini, i quali si accorgono di lei solo quando essa comincia a parlare (10-13), ha radice e motivazione nel terremoto. Ma anche la gran parte della prima fase del canto, in cui Virgilio chiede la ragione del terremoto e Stazio puntualmente risponde (34-72), spiega precisamente l'avvenimento e lo colloca nella storia della peregrinazione di Dante verso la salvezza. Anche all'altro capo, il terminale, del canto si riconosce bene, nel troncamento del colloquio sulla battuta di Stazio dopo il vano tentativo che questi compie di "abbracciar li piedi" di Virgilio ("Ed ei surgendo: "Or puoi la quantitate / comprender de l'amor ch'a te mi scalda, / quand'io dismento nostra vanitate, / trattando l'ombre come cosa salda", 133-36), che l'episodio non è terminato, che ha bisogno d'un seguito e soprattutto d'una spiegazione del rapporto che si deve instaurare tra Virgilio e Stazio. Il quale è subito di comprensione e d'intesa, ancor prima di sapere l'uno dell'altro; il che avviene prima per Stazio nella seconda fase del canto, poi per Virgilio nell'estrema. D'altronde il rapporto Virgilio/Stazio non importa per sé, ma per il senso che assume nella vicenda di Dante pellegrino della salvezza e dunque per il posto che occupa nel complesso dell'opera, simbolo del sistema universale voluto da Dio. Nell'opera/simbolo resta anche compreso il rapporto tra Dante e Stazio, come fin dal principio s'è istituito quello tra Dante e Virgilio; e del resto non si tratta d'altro che di particolari momenti, di diversa rilevanza e d'un diverso grado di rappresentatività, del legame tecnico-letterario, storico-culturale, memoriale-figurale tra Dante e i poeti. Al primo livello della retorica, dunque, il canto 21 del Purgatorio si spiega solo registrandone le interne insufficienze, unicamente colmabili con un infittimento dei legami che lo stringono alle zone circostanti e al tutto.

Al secondo livello della retorica, quello della dispositio ovvero della distribuzione delle parti, che in un'opera narrativa, com'è nel suo primario significato strutturale la Commedia, è di palmare importanza, fin da una prima verifica le risultanze sono di segno del tutto rovesciato. Le fasi che costituiscono il canto sono individuabili senza incertezze nella loro successione. Precede un'apertura ab- 
bastanza lunghetta (1-33) in cui, dopo una enunciazione della condizione interiore di Dante di fronte ad eventi che oltrepassano la sua capacità di comprensione, e anche quella di Virgilio, come si vede di seguito, vien raccontato l'incontro con l'ombra di Stazio come emersa dalle tenebre dei condizionamenti del peccato e della penitenza. Nelle rapide battute del principio il colloquio par stentare ad avviarsi, inciampando nella meraviglia di Stazio alle parole di Virgilio che si dice relegato "ne l'etterno esilio". Questi, che ignora ancora tutto di Stazio, non solo la sua qualità di poeta, ma perfino quella di anima appena svincolata dai tormenti della penitenza, par tuttavia intuire qualcosa, servendosi d'un giro dotto, ampio e solenne di discorso, come se a sua volta volesse suggerire all'ascoltatore Stazio qualcosa di sé. In casi come questo, naturalmente, si può bene formulare l'ipotesi che Dante autore assegni al suo Virgilio, quasi inconsciamente, il livello di discorso che secondo lui gli compete; Virgilio, insomma, parla alto e solenne, perché è poeta. Ma osta a questa ipotesi, apparentemente semplice e quasi ovvia, il ricordo delle molte volte che, lungo tutto il cammino compiuto fin qui nell'oltretomba, ma specialmente lungo l'Inferno, Virgilio ha modellato il proprio linguaggio secondo le occasioni, le situazioni, i personaggi soprattutto che si trovava dinanzi. E se il linguaggio di Virgilio ha nel Purgatorio caratteristiche generali di cortesia confacenti alla qualità dei penitenti, di spiriti cioè che godono d'una assicurata prospettiva di salvezza, quella rotonda solennità, tendente perfino alla ridondanza, è propria solo di certi incontri e colloqui, particolarmente quelli con dotti e poeti, come in questo caso. In cui Virgilio avverte almeno di essere al cospetto di qualcosa di eccezionale e di un personaggio di qualità.

Segue (34-78) il vero e proprio primo tempo del canto, consistente nella domanda di Virgilio sul perché del terremoto, e nella risposta, anche questa articolata, se non eloquente, comunque dotta, di Stazio, che così resta collocato su di un piano alto, perché non illustra semplicemente a Virgilio e per lui a Dante, come generalmente è accaduto finora in incontri con dannati e penitenti, una condizione post mortem o uno stralcio qualsiasi di una vita passata, ma spiega un evento che ha carattere strutturale del mondo ultraterreno, così come Virgilio ha fatto poco prima, nel canto 17 , a proposito della struttura generale del Purgatorio. Lo scambio di battute si conclude col rapido commento conclusivo di Virgilio, cui s'aggancia, senza soluzione, nel seguito della stessa battuta, il secondo tempo del canto che contiene la richiesta a Stazio di dire di sé e la risposta di lui (79102). Per breve che sia, la domanda di Virgilio è doppia: chiede allo spirito chi fu e "perché tanti secoli sia giaciuto" nel cerchio degli avari. Virgilio riprende parole appena pronunciate da Stazio, che ha affermato d'esser "giaciuto a questa doglia / cinquecent'anni e più" (67-68), parole che, poco specificando, tuttavia approssimano gli universi psicologici e storici dei due poeti. Quello che conta però in questa fase di esame della dispositio è che questa seconda parte della domanda non riceve risposta e solo nel canto 22 Stazio spiegherà di essere stato peccatore per prodigalità e non per avarizia. Se, come è giusto, non si vuol troppo speculare su quelle che possono apparire o essere dilazioni o anche piccole 
contraddizioni, basterà richiamarsi ad un principio che largamente governa la Commedia, quello secondo cui nessun momento di essa risponde ad esigenze di verosimiglianza e tanto meno di realismo, perché in essa tutto è finalizzato alla realizzazione d'una compiuta simbologia del cammino verso la salvazione. È questa il vero referente dell'opera ed essa solo giustifica la sterminata fenomenologia dell'essere che ne può essere assunta come funzione.

E veniamo alla risposta di Stazio, tramata dall'evocazione del suo antico tempo, che, come ogni tempo storico, ha la sua ragione e il suo senso nel troncamento che nella storia umana ha prodotto la venuta del Cristo (82-84). Su questo sfondo prendono consistenza il sentimento di pienezza ch'egli prova ora a redenzione raggiunta nel ricordare la sua antica qualità di poeta (85-93) e la riconoscenza verso il maestro di tutti i poeti, Virgilio, del quale si avvia qui (94102) un discorso che avrà compimento solo nel canto successivo, quando Stazio confesserà quale molla sia stata un'altra opera di Virgilio, la quarta ecloga, nel sospingerlo verso la conversione (22.64-73). Allora sarà sanzionato definitivamente il parallelo per ora solo accennato, e perfino oscuramente nel paradosso espresso alla terzina $100-02$,

\author{
E per esser vivuto di là quando \\ visse Virgilio, assentirei un sole \\ più che non deggio al mio uscir di bando,
}

tra accesso alla poesia e conoscenza di Dio. La conclusione del canto (103-36) è occupata da quella piccola commedia degli equivoci che induce un'atmosfera di distensione e di confidenza, di sorriso non privo di un vibrar di commozione: si tratta insomma veramente d'una pausa e non d'un approdo dell'episodio.

Sul carattere comico del quale in questo suo finale c'è stato un contrasto d'opinioni che vale la pena di richiamare per i suoi sottintesi di carattere generale. Sulla necessità di rifiutare un'interpretazione comica di questo finale aveva indugiato Mario Sansone in polemica con chi (D'Ovidio, Porena) vi aveva visto una manifestazione di realismo borghese sotto l'aspetto della quotidianità, della semplicità di gesti e di parole, e con la motivazione che si trattava invece di un clima di umiltà, di un'esultanza che cede alla sospensione, accettando per conclusione il giudizio di Tommaseo che trovava "quel punto di drammatica e di morale bellezza". Giusto il rifiuto di Sansone di vedere qui un effetto di piccolo realismo, ma improprio tradurlo in una sorta di piccolo psicologismo intrinseco alla situazione, perché esso fa perdere quella sorta di conversione rituale che culmina nel tentato abbraccio dei piedi di Virgilio da parte di Stazio. È quello che ha messo in evidenza poi Borsellino, cui la misura di azione comica di questo finale appare giustamente in opposizione all'apertura del canto successivo (muovendosi dunque sull'asse della continuità dei due canti), senza tuttavia apparirgli né casuale né incongrua. E difatti casuale o incongrua non è, perché a livello di inventio questa piccola commedia si collega con la narrazione del viaggio e al riconoscimento del posto che ha in esso la poesia, mentre a livello di di- 
spositio il troncamento del colloquio sulla battuta di Stazio lo è anche della narrazione. La commediola non è insomma casuale né incongrua perché è investita da precise ragioni retoriche che riconducono il suo comico nel solco grande del senso per cui si ha la Commedia, un'opera cioè di fine lieto. Comico, dunque, vale pausa confidente e sorridente, destinata a risolversi poco più avanti quando Stazio illuminerà appieno la sua devozione a Virgilio con l'alta e seria ragione ch'essa ha.

L'opposizione tra i due livelli fondamentali della retorica è accentuata dalle sottili filigrane di rimandi e viceversa dall'intricata rete di troncamenti che gli altri due suoi livelli, quello della sentenza e quello della locuzione, presentano verso luoghi circostanti e luoghi anche lontanissimi della Commedia. Un parlar sentenzioso è intrinseco alla figura di Stazio in conseguenza della sua stessa funzione: che da una parte è di mostrare con la sua apparizione una diversa condizione dell'essere ed è dall'altra di spiegare cose che i suoi interlocutori ignorano. La sua prima risposta a Virgilio circa la causa del terremoto è tutta un po' sentenziosa. Quando poi giunge a delle conclusioni o semplicemente a passaggi dimostrativi obbligati della spiegazione che viene offrendo o anche meglio si concreta in recise asserzioni, questa generale sentenziosità risulta deliberata e marcata. Basti pensare all'enunciazione con cui s'apre la risposta di Stazio intesa a riassumere la realtà fondamentale che governa la montagna del Purgatorio:

... Cosa non è che sanza

ordine senta la religïone

de la montagna, o che sia fuor d'usanza.

All'enunciazione primaria segue di rincalzo, in una tonalità non meno sentenziosa, la conseguenza teorica diretta di essa sul terreno dei fenomeni fisici, uno dei quali è il terremoto:

Libero è qui da ogni alterazione:

di quel che 'l ciel da sé in sé riceve

esser ci puote, e non d'altro, cagione.

Infine, per verifica, ma con abbondante ridondanza, la descrizione delle situazioni che non possono manifestarsi sulle pendici della montagna, tra poco per di più vanificate dalla ripetizione di gran parte di questa argomentazione nelle parole di Matelda (28.88-144): passo di artificiosa abilità anche, come sempre suona ed è il dire per negazione, il definire dichiarando ciò che non può essere, ma anche occasione di sfoggio e di dominio di un'entità letteraria come la perifrasi, una volta derivandola dall'onomastica mitologica, "figlia di Taumante" per Iride (50), e un'altra dalla tradizione neotestamentaria, "vicario di Pietro" (54) per indicare l'angelo che custodisce l'ingresso al vero Purgatorio. Anche se più 
smorzata nella verifica dimostrativa, la sentenziosità continua a snodarsi lungo tutta la dotta, o almeno esperta, risposta di Stazio, come mostrano le terzine centrali di essa:

De la mondizia sol voler fa prova, che, tutto libero a mutar convento, l'alma sorprende, e di voler le giova.

Prima vuol ben, ma non lascia il talento che divina giustizia, contra voglia, come fu al peccar, pone al tormento.

Stazio commenta qui con molta sottigliezza quello che ha appena ora finito di provare in se stesso: la volontà di salire verso la beatitudine dei cieli è la sola necessaria garanzia che permette di realizzare il desiderio; volontà che sempre il penitente ha recato dentro di sé, trattenuta però dall'opposta volontà di soddisfare il debito contratto verso la giustizia divina col peccato commesso. Cosa v'è, in battute come questa, di rinviabile al linguaggio di Stazio poeta così come nella sua opera veramente si realizzò? Non è piuttosto questo il linguaggio della tarda scolastica, con le sue distinzioni, ripetizioni delle stesse parole in una varietà di accezioni, acuminate e angolose strutture sintattiche? Si potrà dire comunque che posto in bocca a Stazio, perfino nella sua sproporzione, ne fa crescere l'immagine di dotto, di poeta la cui dottrina è cresciuta con la sua conoscenza, rendendo per una via singolare ancor più indissociabili poesia e dottrina.

Non estranea al tono della sentenziosità, anche quando la sentenza non si fissa nella vera e propria forma retorica, è la seconda risposta di Stazio. S'apre con la già sottolineata indicazione storica che rinvia alla distruzione di Gerusalemme per determinare la stagione dell'esistenza terrena di un poeta classico:

Nel tempo che 'l buon Tito, con l'aiuto del sommo rege, vendicò le fora ond'uscì il sangue per Giuda venduto ....

È un'apertura singolare, che sul principio può apparire un po' pletorica, non strettamente necessaria, sfoggio dottrinale di un tema che nel Paradiso Dante avrà bisogno di affrontare di petto, enunciato in 6.88-93 e ampiamente svolto in 7.18-93. Ma a guardar bene la funzionalità di essa è bene appoggiata ad almeno due motivazioni rilevanti e tra loro congiunte. Che il poeta latino imprenda a narrare di sé richiamandosi alla giusta vendetta operata contro la "vendetta del peccato antico" (appunto Par. 6.93) apre naturalmente uno spiraglio immediato sulla conoscenza/esperienza della storia sacra, innesta insomma agostinianamente la storia cristiana sulla storia romana: pochi versi sotto, (87), viene la conferma che questo spirito, che si dice di poeta, è stato cristiano. È un cenno rapidissimo, dato 
in forma di negazione, "non con fede ancora", sommerso subito da un contesto fatto tutto di rinvii alla poesia e ai poeti. In primo luogo, nel suo giro solenne, si ricongiunge all'inizio della prima battuta di Virgilio segnato da una eloquenza letteraria che accoglie in densi riferimenti mitologici la designazione di Làchesi:

Ma perché lei che dì e notte fila

non li avea tratta ancora la conocchia

che Cloto impone a ciascuno e compila ... .

Il rapporto/contrasto tra la concezione del destino nella cultura pagana e nella cristiana opera così chiaramente nelle maglie larghe del discorso. Ma nella materialità del discorso i due interlocutori si riconoscono subito come figure di alto parlare, padroni di mezzi espressivi che sono spie della qualità di poeti. Nell'articolazione del colloquio appare semmai singolarmente nuda la seconda domanda di Virgilio (76-81), concentrata in due terzine secche secche, una per registrare l'acquisizione di un sapere maggiore, l'altra per formulare una richiesta d'identità. Ma si sa quanto l'alternanza di toni e di modi alti e bassi sia intrinseca alla pratica retorico-stilistica di Dante, che qui ricorre ad una sorta di larga antitesi, ad un'opposizione che ponga in risalto, di fronte alla circostanziata funzionalità della domanda, la dotta pienezza della risposta, quasi a soddisfare un desiderio di comparire di Stazio, che dentro di sé si sente discepolo di quel Virgilio che, senza ch'egli lo sappia, lo ha interrogato.

L'avvio della seconda risposta di Stazio è dunque tutt'altro che pletorico; serve invece puntualmente a mostrare dall'interno del linguaggio le implicazioni, i sottintesi ancora da svelare del colloquio tra Stazio e Virgilio, illuminati poi nella piccola commedia conclusiva del canto e ampiamente nel canto successivo quando questo richiamo alla storia sub specie christianitatis viene spiegato dalla narrazione distesa della conversione di Stazio. Serve soprattutto a inquadrare la presenza di Stazio nell'ambito degli incontri coi poeti e insieme sotto il segno della concezione cristiana della storia. Sta in questo andamento l'aria di sentenziosità che dalla prima terzina si diffonde sull'intera risposta e si concreta nell'indicazione dell'Eneide quale "divina fiamma / onde sono allumati più di mille" (95-96).

Al parlar sentenzioso fa da sostegno la consistente ricercatezza dell'elocuzione a tutti i livelli dell'organizzazione linguistica. Si tratta di una ricercatezza che, piuttosto che sull'eleganza delle scelte lessicali, si fonda sullo spessore semantico di esse, accresciuto anche dalle valenze metaforiche, sovente e notevolmente ampie. Su alcuni elementi la critica ha scavato con profitto: Luiselli ha esemplarmente ricostruito per l'"acqua" del v. 2 il complesso spessore semantico, decifrando la stratificazione di significato simbolico che il termine ha avuto per tutta la cristianità principalmente come rinvio alla dottrina rivelata e allo Spirito Santo, con una tendenza di ciascuno dei due significati ad implicare l'altro, e giungendo a ripuntualizzare in funzione di questo canto e di questo luogo rile- 
vazioni che non si era mancato di fare in passato circa anteriori riflessioni di Dante in proposito specialmente nel Convivio. Nel richiamare il gran lavoro di scavo compiuto intorno al lessico, mi fermo su questo termine, tanto emblematico in sé proprio ad apertura di canto, non solo per gli approfondimenti cui ha invogliato e $\mathrm{i}$ dati che ha consentito di accumulare, ma perché da esso e da questo lavorio si deduce il senso complessivo dell'episodio di Stazio come tessera del cammino di Dante verso la conoscenza della verità rivelata in un continuo scambio tra momento razionale e momento mistico dell'atto della conoscenza, come giustamente ha sottolineato Padoan, in una prospettiva generale della gnoseologia dantesca. Dai metodi di lettura praticati da Padoan e da Luiselli scaturisce il senso di quanto conti il singolo termine, l'elemento microstrutturale nella proiezione di fatti più complessi e globali, macrostrutturali, in grado di condizionare lo svolgimento di un episodio e di collegarlo al senso generale del poema e al rapporto tra esso e la tradizione culturale cui si richiama.

Per brevità mi fermerd solo su alcuni termini che nel loro spessore semantico rivelano l'importanza che questo canto riveste nel congiungimento di poesia e conoscenza nei suoi vari gradi. "Acqua" è risposta a "sete natural": questo sintagma è metafora di ansia di sapere, di desiderio di conoscenza, un fattore che governa da sempre la mente di Dante e, in generale, sia pure in modi differenti, di tutti gli uomini, "fatti ... non a viver come bruti / ma per seguir virtute e canoscenza" (Inf. 26.119-20), luogo obbligato della gnoseologia dantesca che motiva i frequenti accostamenti che nelle vicende critiche del 21 del Purgatorio si sono avuti tra Stazio ed Ulisse. Questa "sete", prima parola del canto è rienunciata altre due volte nel suo corso, prima per la speranza che Virgilio con la sua domanda suscita nell'animo di Dante:

.. pur con la speranza
si fece la mia sete men digiuna;

- e in questa speranza che allenta il desiderio s'inciampa in qualcosa d'artefatto, velato solo dall'attitudine di Dante a sottolineare il valore gnoseologico del canto attraverso l'intensa collaborazione, spesa a suo beneficio, tra Virgilio e Stazio; poi a staccare la prima dalla seconda fase del canto, ma anche qui in un contesto non sgombro d'un che d'artefatto, un po' pleonastico e persino non precisissimo di senso:

... e però ch'el si gode

tanto del ber quant'è grande la sete,

non saprei dir quant'el mi fece prode.

Dove non può sfuggire l'ambiguità o debolezza del sintagma "non saprei dir" anche se interpretato come ineffabilità ("è tanto grande il piacere di aver bevuto, 
che non so dirlo"). A conferma della funzione gnoseologica del canto sovviene anche la frase conclusiva del canto precedente che offre un suggerimento circostanziato all'apertura del 21 , come poche altre volte avviene in tutta la Commedia:

Nulla ignoranza mai con tanta guerra

mi fé disideroso di sapere, se la memoria mia in ciò non erra,

quanta pareami allor, pensando, avere; né per la fretta dimandare er'oso, né per me lì potea cosa vedere:

così m'andava timido e pensoso.

È un passo estremamente rilevante, perfin nella e a causa della sua disadorna semplicità, per l'atteggiamento interiore e i comportamenti esteriori di Dante di fronte a cose e persone che incontra e a eventi cui assiste. L'espressione "disideroso di sapere" è precisa anticipazione di "sete natural", opposta a "ignoranza", completata, nel suo irritante senso di debolezza e impossibilità, dal v. 150, "né per me lì potea cosa vedere" (ero insomma incapace di capire da solo cosa avveniva), fatta più acuta da quel camminare in fretta fra $i$ corpi degli avari che assiepano lo spazio della cornice. Questa rete di rinvii alla conoscenza come desiderio e "sete natural" è richiamata ulteriormente attraverso un fiorire di espressioni vagamente metaforiche quali "la cruna / del mio desio" di 37-38, che ribadisce il senso della "sete natural", e "li occhi miei" di 124 , che è puramente metafora di conoscenza.

Tutto il canto è percorso da interni rimandi e ripetizioni lessicali e concettuali, da espressioni singolarmente pregnanti nella loro concentrazione semantica: si vedano forme come "la 'mpacciata via" (5), il cammino ostacolato dai simulacri degli avari sparsamente distesi; "la sepulcral buca" (9) per indicare il santo sepolcro secondo la testimonianza concorde di Matteo (27.60), Marco (15.46), Luca (23.53) nel senso di "tomba scavata nella roccia"; "beato concilio" (16), distinto da "verace corte" (17) e opposto ad "eterno esilio" (18), nella stessa terzina e in rima; "l'ampia gola / d'inferno" (31-32) con il doppio valore fisi$\mathrm{co} /$ morale dell'aggettivo; "secco vapor" (52), termine tecnico, il vento che, secondo la teoria aristotelica, genera le scosse terrestri; "mio vocale spirto" (88) per poesia; "seconda soma" (93) per designare l'Achilleide dopo la Tebaide; e si potrebbe continuare. Quanto ai rimandi, si guardi a come "giusta vendetta" di v. 6 , che semplicemente allude alla punizione degli avari, giusta, com'è in generale giusta ogni penitenza scontata da un peccatore, lo è anche per il contrappasso, ma insieme anticipa la ben più inglobante vendetta di Tito contro gli uccisori di Cristo di 82-84, di cui si son dette le conseguenze di Paradiso 6 e 7 . Tutto questo evidenzia la fermezza concettuale, ideologica del disegno nel quale Dante iscrive anche gli elementi infinitesimali che compongono il tessuto del canto. Sul terre- 
no delle ripetizioni o riprese, che sono anche variazioni e ampliamenti di significato si va da elementi minuscoli come la particella avverbiale "ci" che s'incontra quattro volte, ai vv. $10,45,58$ e 78 , e che induce a dar rilievo al senso di hic et nunc che Dante intende imprimere alla situazione, a elementi di significato ampio come "sete" di cui s'è detto, come "voler" nella doppia funzione di sostantivo e di verbo rispettivamente a 61 e 63, collegato con la nozione di libertà mediante l'aggettivo "libero" di v. 62 , già usato al v. 43 con allusione alla libertà da servitù terrene di tutta la montagna.

A proposito di elocutio non si può tacere della frequenza di certe figure: si è accennato alle perifrasi dei vv. 50 e 54 come casi di ostentazione di letterarietà, ma perifrastico è anche tutto l'inizio del canto e in forma perifrastica è presentata Làchesi in 25-27, perifrasi inserita in un'altra più larga per dire che Dante è vivo; e perifrastica, come s'è visto, è l'indicazione del proprio tempo storico data da Stazio (82-83). Segue la più esaltante delle perifrasi del canto, quella per poeta, "il nome che più dura e più onora" (85). Lampanti le implicazioni metaforiche di alcune di queste perifrasi, che s'inquadrano in un sistema espressivo che presenta un livello metaforico singolarmente alto e teso lungo tutto il canto. Non insisto perché per la maggior parte sono già emerse nel corso di questo esame del canto sotto il profilo dell'opus retoricum. Il quale, proprio attraverso questo varco della metafora, passa la mano all'opus symbolicum; al di là delle contingenze della mediazione tra organizzazione del discorso (o retorica) e sovrasenso del discorso (o simbolo) va insomma riconosciuto l'intreccio permanente tra opus retoricum e opus symbolicum. Questo non solo ha nella retorica il mezzo di manifestazione e gli strumenti dell'esprimersi, ma si annida nel cuore di questi mezzi e strumenti, non solo si identifica in essi, ma li genera, li giustifica e li destina.

In quest'ordine va colto e di volta in volta isolato il definirsi del simbolo, nella sua varia e molteplice consistenza ed entità, da elementi microstrutturali, che possono essere una frase, un'immagine, una figura o anche solo una parola, e possono manifestarsi a livello implicito, a fattori macrostrutturali, come eventi, occasioni di discorsi, discorsi in se stessi, personaggi che li tengono e personaggi d'ogni genere. Fra questi, ovviamente, il primo posto spetta a Stazio, sul cui significato complessivo nella Commedia come "anima redenta, giunta alla fine dell'espiazione, già pronta a salire al cielo e perciò atta ad assolvere anche il compito di addottrinare Dante in un arduo punto della scienza teologica, come avviene nel canto XXV" (Paratore 5: 422, I colonna),-dove la domanda di Dante su "come si può far magro / là dove l'uopo di nodrir non tocca" (25.20-21) è soddisfatta largamente con l'illustrazione dell'intero rapporto tra anima e corpo dal loro costituirsi in unione e separarsi-ha dato un nitido quadro riassuntivo Paratore nella voce "Stazio" dell'Enciclopedia dantesca, cui non si pud che rinviare anche per la puntuale elencazione di quali elementi di linguaggio, di informazione, di poetica pervenivano a Dante da Stazio.

Di questo cammino accidentato dei possibili significati di Stazio, avviato già da Pietro di Dante che vedeva simboleggiata in Stazio la filosofia morale, basterà 
ricordare qualche momento, magari ponendosi un po' al di là della prospettiva accolta da Paratore, secondo cui "l'intervento dottrinale di Stazio nel canto XXV è stato sempre considerato la chiave per spiegare la funzione di Stazio nei canti del poema in cui egli appare" (Paratore, ibidem). Per esempio non sarà più da trascurare l'interpretazione di Stazio come figura Christi proposta da Padoan anche al di là delle motivazioni euristiche da lui addotte, ma semmai, valendosi dell'interpretazione, che pare più ovvia, di Bontempelli, secondo il quale nell'episodio staziano del canto 21 del Purgatorio è da vedere esplicitata la trattazione delle modalità secondo cui un'anima si libera dalla condizione penitenziale, riconoscere in Stazio un'anticipazione dell'esperienza stessa di Dante, una figura Dantis, se si può dire, che è a sua volta figura Christi, almeno nella misura in cui ogni cristiano si riconosce nella vicenda della passione e della morte di Cristo, e quindi della resurrezione e della salvezza. Allo stesso modo la tesi che fu principalmente di Renucci e di Ronconi, secondo la quale lo Stazio dantesco è un'invenzione funzionale all'esaltazione di Virgilio, andrà accolta tenendo conto della molteplice simbolicità che hanno sempre le figure dantesche; le quali neppure perdono mai un qualche rapporto con l'universo che per Dante, come per tutta la cultura cristiana tardo-antica e medievale, è la realtà, cioè l'insieme di accadimenti storici, fatti naturali, eventi sovrannaturali, strutture metafisiche che s'inseriscono nel percorso che sta tra la causa efficiente e la causa finale, percorso apparente che muove da e riconduce a Dio creatore.

Lo Stazio del canto 21 , che parla di sé sotto il segno della concezione cristiana, ovvero neoplatonica-agostiniana, della storia, descrive come un'anima libera dal Purgatorio sale al cielo, si lascia implicare nella commedia degli equivoci della conclusione del canto, è, tutto insieme, il poeta, il dotto, l'anima redenta che è per Dante modello e anticipazione, ma anche l'"anima semplicetta", rinnovellata, restituita alla sua infanzia, "a guisa di fanciulla / che piangendo e ridendo pargoleggia" (16.86-87), seppure ormai separata per sempre dall'ignoranza del bene e di Dio. Stazio ha dunque un alto livello di simbolicità, manifesto nei suoi due interventi, ma anche nella sua apparizione e presenza.

L'altro elemento di alta simbolicità è il terremoto che, con particolareggiata tematica, collega il canto 20 al 21 . Annunciato sotto il segno d'una mitologia che può parere perfino un po' stravagante:

Certo non si scoteo sì forte Delo pria che Latona in lei facesse 'l nido a parturir li due occhi del cielo;

ma che si allinea con quanto abbiamo detto sull'intreccio di paganità e cristianità, è coronato subito dal grido neotestamentario dei pastori ( $L c .2 .14)$ ripetuto dalle anime sparse per tutte le cornici. Qui il ruolo dei pastori estatici è assolto da Virgilio e da Dante, che assistono al terremoto in condizione di ignoranza e desiderano sopra ogni cosa sconfiggerla. Si trapassa così da una suggestione mitolo- 
gica ad una dimensione biblica, che bene si spiega nell'economia dell'interpretazione "scritturale" della Commedia, la quale come poema sacro è una trascrittura, continuazione della Sacra Scrittura. E in questo senso si può ben sottolineare che la referenza dantesca per la fonte di questo terremoto è quell'altro terremoto che nel momento in cui Cristo spira scuote la terra e spacca l'arca, come è raccontato in Matteo 27.50-54: "E Gesù, avendo di nuovo gridato con gran voce, rendé lo spirito. Ed ecco, la cortina del tempio si squarciò in due, da cima a fondo, e la terra tremò, e le rocce si schiantarono, e le tombe s'aprirono, e molti corpi de' santi che dormivano, risuscitarono; ed usciti dai sepolcri dopo la risurrezione di lui, entrarono nella santa città, ed apparvero a molti". Tra l'evento del terremoto com'è descritto sulla fine del canto 20 e le spiegazioni che nel 21 Stazio, subito dopo essere apparso ai due viandanti, dà sulla sua causa, sembra che Dante voglia costituire una sorta di saldatura tra l'evento della nascita di Cristo e quello della sua morte; nascita e morte dunque di un'anima, dove però morte significa solo rinascita, raggiungimento di una nuova vita, più alta, approdata alla salvezza. Così risulta che le spiegazioni di Stazio circa i modi di liberazione di un'anima dal Purgatorio sono premesse e condizionamenti stringenti per quello ch'egli spiegherà poi, dopo l'intermezzo dell'incontro con gli altri due poeti, Forese e Bonagiunta, nel canto 25. Che non si può assumere dunque come illustrazione della funzione di Stazio negli episodi che lo riguardano, perché questa funzione è molto più articolata e si dica pure complessa e variata. Modello dunque Stazio di un particolare modo di manifestare in quanto simbolo, attuato con precise modalità e strumentazioni retoriche, la sola e unica verità del poema: il cammino verso la redenzione.

In tale cammino tutti e tre i canti staziani con le loro dimostrazioni, le spiegazioni e le teorizzazioni in essi contenute hanno un loro senso, che ne fa delle precise tappe del viaggio intrapreso: ed è grave e grossolano errore romanticoidealistico aver considerato questi apparati dottrinali talvolta come vani indugi, tanto nella prospettiva dell'opus symbolicum, poiché la redenzione premerebbe per altre vie più rapide e di significazione più immediata, quanto in quella dell'opus retoricum, perché per definizione la retorica sarebbe un impedimento della poesia. Per Dante, al contrario, i processi simbolici come i processi retorici sono lenti e complessi, non essendo altro che gradi di accesso al sapere e alla spiritualità. In questa direzione il canto 21 , con tutti i legami micro e macrostrutturali che ha con la cantica e con l'intero poema, è una premessa di sviluppi e un modello di percorsi discorsivi: lo è in generale come prima definizione di funzione della poesia che sarà perfezionata specialmente in 24 e 26 da Bonagiunta e da Guinizzelli per un verso, quello stilistico-linguistico, e 23-25 per altro verso, quello dottrinale morale (quest'ultimo con una punta personale che rende individualissimo l'incontro con Forese), e lo è in particolare per il canto 22 per la valenza simbolico-retorica di esso.

Appena, infatti, entrati nel "sesto giro" (22.2) che è dei golosi, Virgilio muove la sua continuata inchiesta sulla persora di Stazio, la sua storia, i suoi motivi, il 
suo destino: e si ha la prima domanda circa la creduta avarizia di lui (10-24) seguita dall'articolata risposta di Stazio che rovescia in peccato di prodigalità l'avarizia (27-54). Dove cio che importa non è certamente il ristabilimento di una verità terrena, ormai tutta da dimenticare da parte di Stazio (e di scarsa pregnanza per il progresso morale-spirituale di Dante), ma il ritorno al tema della poesia e dell'esser poeti. Son le notizie recategli da Giovenale, dunque, a inclinare benevolmente l'animo di Virgilio verso Stazio senza averlo conosciuto (1317) e sono i versi virgiliani di Aen. 3.56-57 (volontariamente o involontariamente fraintesi, come la larga e antica tradizione critica ha voluto, oppure latamente intesi, come oggi si vuole, quale denuncia del "principio e causa comune di entrambi gli eccessi, degli avari e dei prodighi": vedi per tutti Sapegno, commento al luogo) a governare la risposta di Stazio (64-99). L'universo della poesia, nei suoi rappresentanti e nei suoi interpreti, consente dunque di oltrepassare le soglie del cammino verso la redenzione e la verità.

Incalzante la seconda domanda di Virgilio circa il Cristianesimo di Stazio (55-63) e altrettanto lunga e articolata che la prima la seconda risposta di Stazio (64-99). La bibliografia accumulata sul tema è, come si sa troppo bene, sterminata e anche sostanzialmente divisa tra chi ha cercato le fonti disponibili a Dante affinché egli accogliesse questa convinzione e chi invece ha guardato ad individuare l'impegno dantesco a cristianizzare Stazio in funzione della sua collocazione nella Commedia. Anche se fosse utile, non sarebbe in questa sede possibile disegnare le vicende e le ragioni di questa discussione o insomma varietà di interpretazioni, che, del resto, sarebbe inutile riassumere senza mostrare in piena luce le motivazioni e le argomentazioni addotte in un senso o nell'altro. Come è accaduto per tutte o almeno tante altre cruces della poesia di tutti i tempi, erudizione e filosofia hanno collaborato ad allargare l'ermeneutica possibile del luogo dantesco in maniere perfino avulse dal testo, magari nella goffa ansia di decifrare allusioni che invece per Dante erano chiarissime dichiarazioni.

Ma sia come sia, una cosa almeno è certa: di tutto il lungo rapporto tra Virgilio e Stazio, all'interno del quale Dante è come messo da parte, insomma viene a trovarsi in una posizione marginale come accade allo scolaro quando due maestri discorrono o disputano tra di loro, questo è il momento culminante. Uno Stazio cristiano e poeta, che innalza Virgilio a suo maestro morale e letterario valendosi di una ermeneutica solidamente radicata nella cultura medievale, illumina e spiega distesamente e profondamente il rapporto tra poesia e cristianesimo e dunque tra Virgilio e Dante stesso. Pare anzi che la retorica stessa si proietti qui nel suo significato simbolico correlato: e il calore col quale Dante rende la sua nuova e alta testimonianza di fedele di Virgilio colloca questa tra le più magnifiche e tout court poetiche ch'egli abbia pronunciato:

\section{... Tu prima m'invïasti}

verso Parnaso a ber ne le sue grotte, e prima appresso Dio m'alluminasti.

Facesti come quei che va di notte, 
che porta il lume dietro e sé non giova, ma dopo sé fa le persone dotte,

quando dicesti: "Secol si rinova; toma giustizia e primo tempo umano, e progenie scende da ciel nova".

Di fronte a questa esaltazione le altre cose, la compassione per i martiri, la conversione, il nicomedismo di Stazio passano in secondo piano. Ciò che conta è quel linguaggio preumanistico che salda la tradizione antica con la moderna, la precristiana con la cristiana, che rende unitaria la storia umana e che esalta la funzione della parola e particolarmente della parola poetica. (Di seguito, andranno rilevati i vv. 79-80: "E la parola tua sopra toccata [la citazione cioè della quarta ecloga] / si consonava a' nuovi predicanti".)

Imboccata questa via, dello stato poetico_di una poesia da intendere come premessa, avvio al sapere-quale valida condizione di accostamento alla fede diventa confidenzialmente legittima l'ansiosa richiesta di Stazio, che da interrogato si fa interrogante, sul destino ultraterreno degli antichi poeti latini e greci, che, magari poco o niente conosciuti da Dante, sono stati ben noti a Stazio e a Virgilio (97-99). Ovviamente, la ripresa di Inferno 4 implica un ricalco anche contenutistico e così la risposta rapida di Virgilio comprende insieme autori e personaggi.

A questo punto può riprendere la narrazione e cioè il movimento concreto del viaggio, del cammino verso la redenzione e la salvezza, mentre tuttavia i due poeti antichi continuano a scambiarsi i "lor sermoni" e le loro "dolci ragioni". È stato un vano indugiare del viaggio-narrazione questa complessa illustrazione di sé che Stazio ha offerto a Virgilio per vantaggio di Dante? Senza dire che il primo intervento di Stazio sul terremoto è dottrinalmente funzionale alla natura della montagna del Purgatorio, è abbastanza ovvio cogliere nell'approfondimento della personalità e della storia di Stazio il risvolto interiore dell'esperienza dantesca dell'oltretomba: ma ciò che qui risalta meglio, magari, che altrove è l'unità retorico-simbolica dell'opera e, per conseguenza, la dichiarata, esaltante funzione della poesia.

Semmai, su questo piano più elementare e meccanico si potrà sottolineare fortemente la perfetta reduplicazione di struttura che si ha tra $i$ canti 21 e 22 : dove alle due domande e alle due risposte segue, come un'appendice, che in 21 è il riconoscimento di Virgilio e in 22 è, appunto, la ripresa del cammino, l'improvvisa comparsa dell'albero-immagine di punizione dei golosi e, infine, le voci di esempi di sobrietà che da esso discendono. Piccolo elemento in più di collaborazione ermeneutica per individuare la struttura del dittico.

La presenza di Stazio, appena segnalata in 23.131-33, con richiamo al terremoto e cioè alla comparsa di lui, e in 24.98-99 e 119-20, solo come compagno dei due viandanti, campeggia nuovamente e definitivamente in 25 . Stazio si sostituisce qui a Virgilio per invito di questi (pur apparendo sicuro ch'egli è bene 
informato circa la natura delle ombre dell'oltretomba). Certo è che la finale spiegazione dottrinale di Stazio, dopo quelle intramezzate e da lui udite di Forese e di Bonagiunta, chiude un cerchio apertosi col canto 21. Stazio dà qui tutto quello che ancora può dare (e narrativamente è forse accettabile la proposta sovente avanzata che Stazio prepari il prossimo abbandono di Dante da parte di Virgilio-canto 30-proprio anche con la rinuncia di quest'ultimo a illustrare egli stesso un argomento che conosce). La trattazione dottrinale richiede ovviamente un mutamento immediato di linguaggio, anche se gli agganci con quanto s'è detto ed è avvenuto implicano delle mediazioni e dei rallentamenti. (Uno, vistoso, è subito offerto da Virgilio col rinvio all'esempio di Meleagro come possibile riflessione di Dante sull'oggetto della sua domanda.) Ma i vv. 2-3, col loro ricorso al preciso momento astronomico del tempo in cui avviene il passaggio dal settimo all'ottavo giro, anticipano tale mutamento:

ché 'l sole avea il cerchio di merigge

lasciato al Tauro e la notte allo Scorpio.

E mentre il viaggio a tre continua, Dante è mosso da una curiosità che la pena dei golosi gli ha suscitato:

... Come si può far magro

là dove l'uopo di nodrir non tocca?

La domanda appare ed è in grande misura determinata dalla contingenza dell'esperienza che Dante ha appena compiuto. Ma nella prospettiva generale del poema è molto più rilevante di tale contingenza: e la complessità della risposta ch'essa induce lo dimostrerà con pienezza. Essa infatti ha alle spalle tutta una serie di piccoli e meno piccoli accadimenti, di casi, di gesti che attraverso Inferno e Purgatorio hanno pungolato la mente del lettore con interrogativi non risolti lì per lì, dati come per scontati e, invece, al tirar delle somme o anche solo ad un certo punto delle vicende, affatto scontati. Sono, come dire, in senso letterale, la fisica e la metafisica dell'oltretomba ad essere investite: sono cioè le pene che i dannati e i penitenti soffrono come se fossero corpi viventi, sono le paure fisiche di Dante che sono apparse nel loro concreto accadere sciolte dal loro significato simbolico: sono gli stupori di Dante stesso e di tanti personaggi nell'occasione di gesti che hanno risultati opposti, come è dell'abbracciarsi di Virgilio e Sordello (Purg. 6.75) e l'impossibilità del contatto tra Virgilio e Stazio (Purg. 21.132). È insomma la struttura, la natura anzi dell'intero oltretomba nel suo apparire a Dante in figure concrete a richiedere una spiegazione generale, comprensiva di molte e varie eventualità che appartengono al passato ma in parte, come conseguenze, al futuro prossimo del viaggio.

Dopo il defilarsi di Virgilio (22-30), la spiegazione di Stazio fluisce senza intoppi, lungamente, distesamente occupando quasi tutto il canto (34-108). Che 
cosa v'è di peculiarmente staziano in quella articolata dimostrazione della "teoria delle ombre" che assume una funzione circostanziata per molti luoghi di Inferno e di Purgatorio dove le anime soffrono corporalmente una pena in assenza, cosa che per l'appunto non tocca Stazio libero ormai da ogni pena e avviato sull'ultimissimo tratto della via della salvezza? Perché Dante, per spiegare la consistenza delle ombre, deve rifarsi indietro, al momento in cui l'anima entra nel feto, impostando ancora una volta un problema che la cultura medievale aveva partecipato appassionatamente, ma per il quale egli non pare che abbia soluzioni nuove da avanzare?

Per queste e consimili domande una sola risposta è valida: la Commedia, come universo alternativo, proprio nella sua qualità di struttura simbolica e discorsiva insieme, deve offrire da una parte una spiegazione a tutti gli interrogativi che l'uomo si può porre, insomma ha da presentarsi come sistema filosofico completo ed organico, e dunque anche il problema dell'entrata dell'anima nel corpo deve avere un suo posto; dall'altra, deve per se stessa essere un organismo autonomo e, per quanto possibile, privo di vuoti e anche di contraddizioni. È in questa prospettiva che va vista la spiegazione di Stazio a Dante di questo canto. Lo Stazio della storia non c'entra: conta unicamente lo Stazio personaggio e in quanto tale poeta e cristiano, che quindi può discutere di un problema grave della cultura del tempo di Dante e in generale medievale, può propendere per le tesi elaborate in proposito da Alberto Magno e del tutto conformi alle posizioni tomistiche, pud accogliere di queste la polemica antiaverroistica sull'intelletto possibile e può infine esporre la teoria delle ombre che condensano intorno a sé l'aria dando a questa la forma che l'anima aveva quando era unita al corpo.

Certo, nel lungo discorso di Stazio, che è una dimostrazione filosofica al modo di altre che si sono incontrate anche nel Purgatorio, come quella, prestigiosissima, sui due poteri preposti al governo del mondo esposta da Marco Lombardo nel canto 16, vi sono dei momenti di densa commozione/partecipazione morale/intellettuale, come si palesa nei vv. 67-75, estaticamente contemplanti il formarsi definitivo della persona umana nel corpo e nell'anima:

Apri a la verità che viene il petto; e sappi che, sì tosto come al feto l'articular del cerebro è perfetto,

lo motor primo a lui si volge lieto sovra tant'arte di natura, e spira spirito novo, di vertù repleto, che ciò che trova attivo quivi, tira in sua sustanzia, e fassi un'alma sola, che vive e sente e sé in sé rigira.

Verbum caro factum est: la sacralità dell'atto si mescola con la gioia della vita partecipata nella sua unità che redime la carne e la fa parte dello spirito: "fassi un'alma sola". E tuttavia questa commozione è tutta lucidamente vissuta: non va 
veduta, non può esserlo come un varco alla prevalente dottrinalità del discorso e del canto: è invece un passaggio denso, pregnante della costruzione che essi sono per sé e come conseguenza della destinazione che hanno nel poema, nella realtà di esso o, sarebbe forse meglio dire, nella sua metarealtà. Sicché, come questo luogo è determinante per le fasi successive del presente discorso (poiché, formata l'unità dell'anima e del corpo, la forma di questo resta impressa nell'altra e quindi, al momento della morte, "quando [cioè] Làchesis non ha più del lino", "solvesi da la carne, e in virtute / ne porta seco e l' umano e il divino", tanto che, giunta l'anima nel luogo della sua pena, "l'aere vicin quivi si mette / e in quella forma ch 'è in lui suggella / virtüalmente l'alma che ristette", 94-96), ugualmente determinante esso è per la definizione dell'universo della Commedia.

$\grave{E}$ insomma lo Stazio personaggio dantesco che svolge questa dimostrazione: e la svolge dall'alto della sua qualità di anima prossima al raggiungimento della beatitudine. Egli parla dunque un linguaggio che sarebbe quello di Dante stesso, se solo si confermasse la natura figurale di Stazio rispetto a Dante o, detto altrimenti, con intuizione che fu già di Montanari, Stazio non è che una proiezione autobiografica di Dante (e di ognuno che aspiri a compiere il cammino della salvazione). Proprio per questo, in conclusione, hanno tutto il loro spicco le tonalità più confidenziali e domestiche di discorso che si possono incontrare, consentite appunto ad un compagno di viaggio col quale si ha una comune destinazione:

Quindi parliamo e quindi ridiam noi;

quindi facciam le lacrime e' sospiri che per lo monte aver sentiti puoi.

Secondo che ci affiggono i disiri e li altri affetti, l'ombra si figura; e questa è la cagion di che tu miri.

Chiusa la dimostrazione, con una scansione che abbiamo già rilevato nelle zone finali dei canti 21 e 22, riprende la narrazione del viaggio e cioè il movimento che si svolge ormai sul terreno dell'ultima cornice, che è dei lussuriosi immersi nella fiamma che "la ripa ... in fuor balestra". E saranno ancora poeti a proporsi come penitenti esemplari, Guinizzelli e Daniel, ultimi in buon punto di una successione che per sette canti è tutta di poeti, cioè di figure che si salvano sì per fede, ma che la via della fede imboccano solo percorrendo il cammino della poesia." 
NOTE

* I contributi cui si è fatto riferimento nel testo sono in generale distesamente menzionati nella voce con bibliografia che Ettore Paratore ha steso su Stazio nell'Enciclopedia dantesca (5: 419-25), oggi indispensabile punto di partenza per qualsiasi indagine su Stazio, comprese quelle, qui del tutto escluse, circa le influenze letterarie-stilistiche di Stazio su Dante: argomento che richiede una trattazione specifica estranea a questi contomi. Ad esse, voce e bibliografia, rinvio pertanto e qui mi limito a indicare alcune voci bibliografiche sullo Stazio dantesco successive o non registrate nella voce di Paratore.

\section{OPERE CITATE}

Borsellino, Nino. "Il canto XXII". Purgatorio. Roma: Bonacci-Casa di Dante, 1981. 513-32.

Luiselli, Bruno. "Il canto XXI". Purgatorio. Roma: Bonacci-Casa di Dante, 1981. 487-512.

Montanari, Fausto. L'esperienza poetica di Dante. Firenze: Le Monnier, 1959.

Paratore, Ettore. "Stazio". Enciclopedia dantesca. Vol. 5. Roma: Istituto dell'Enciclopedia Italiana, 1976. 419-25.

Renucci, Paul. Dante disciple et juge du monde gréco-latin. Paris: Société d'édition les Belles Lettres, 1954. 